\title{
Diminished autonomic neurocardiac function in patients with generalized anxiety disorder
}

This article was published in the following Dove Press journal:

Neuropsychiatric Disease and Treatment

8 December 2016

Number of times this article has been viewed

\author{
Kyungwook Kim' \\ Seul Lee ${ }^{2}$ \\ Jong-Hoon $\mathrm{Kim}^{1-3}$ \\ 'Gachon University School of \\ Medicine, ${ }^{2}$ Department of Psychiatry, \\ Gil Medical Center, Gachon University \\ School of Medicine, Gachon \\ University, ${ }^{3}$ Neuroscience Research \\ Institute, Gachon University, Incheon, \\ Republic of Korea
}

\begin{abstract}
Background: Generalized anxiety disorder (GAD) is a chronic and highly prevalent disorder that is characterized by a number of autonomic nervous system symptoms. The purpose of this study was to investigate the linear and nonlinear complexity measures of heart rate variability (HRV), measuring autonomic regulation, and to evaluate the relationship between HRV parameters and the severity of anxiety, in medication-free patients with GAD.

Methods: Assessments of linear and nonlinear complexity measures of HRV were performed in 42 medication-free patients with GAD and 50 healthy control subjects. In addition, the severity of anxiety symptoms was assessed using the State-Trait Anxiety Inventory and Beck Anxiety Inventory. The values of the HRV measures of the groups were compared, and the correlations between the HRV measures and the severity of anxiety symptoms were assessed.

Results: The GAD group showed significantly lower standard deviation of RR intervals and the square root of the mean squared differences of successive normal sinus intervals values compared to the control group $(P<0.01)$. The approximate entropy value, which is a nonlinear complexity indicator, was also significantly lower in the patient group than in the control group $(P<0.01)$. In correlation analysis, there were no significant correlations between HRV parameters and the severity of anxiety symptoms.

Conclusion: The present study indicates that GAD is significantly associated with reduced HRV, suggesting that autonomic neurocardiac integrity is substantially impaired in patients with GAD. Future prospective studies are required to investigate the effects of pharmacological or non-pharmacological treatment on neuroautonomic modulation in patients with GAD.
\end{abstract}

Keywords: generalized anxiety disorder, heart rate variability, parasympathetic modulation

\section{Introduction}

Generalized anxiety disorder (GAD) is a chronic and highly prevalent disorder that is characterized by uncontrollable excessive worry, chronic anxiety, and tension. ${ }^{1}$ Typically, GAD is accompanied by a number of physical symptoms that result in significant impairment of daily social and occupational functioning. ${ }^{2,3}$ In addition to various cognitive and emotional anxiety symptoms, it is further characterized by autonomic nervous system (ANS) symptoms, including hot flashes, palpitations, perspiration, and shaking. ${ }^{2-4}$ The increased cardiovascular morbidity associated with GAD may in fact be related to the ANS dysregulation underlying the disorder. ${ }^{5,6}$

The analysis of heart rate variability (HRV), that is, the variation in the cardiac interbeat interval over time, has proven to be both practical and useful in evaluating ANS dysregulation, since its measurement is noninvasive, easy to perform, and reproducible. ${ }^{7-9}$ Recently, novel nonlinear measures of HRV gave rise to a new approach to the understanding of the complex phenomena surrounding neurocardiac processes and have provided clinically useful information regarding the hidden 
dynamics of ANS dysregulation in neurological and psychiatric disorders. ${ }^{10-14}$

Several studies have reported that there are diminished HRV in patients with GAD, ${ }^{15-19}$ though not all studies have observed such associations; hence, there are few inconsistencies. A recent meta-analysis by Chalmers et al ${ }^{9}$ revealed that the frequency domain measure of HRV, such as high frequency, is significantly reduced in patients with GAD compared to healthy control subjects. This meta-analysis suggests that GAD adversely affects the autonomic regulation of cardiovascular homeostasis, particularly highlighting the adverse effects on the reactivity of the parasympathetic nervous system. ${ }^{9}$

However, several key features of the relationship between GAD and HRV are still unclear. Previous studies ${ }^{15-19}$ on HRV in patients with GAD mainly focused on the linear measures of HRV, especially the frequency domain measures. However, the effects on parasympathetic nervous system reactivity may be better reflected in the time domain measures of HRV than in the frequency domain measures alone. ${ }^{9}$ In addition, the relationship between the HRV measures and the severity of anxiety in patients with GAD has not been sufficiently investigated and needs further exploration. Moreover, the nonlinear dynamic measures of HRV have not been explored so far in GAD. Even though the nonlinear measures of HRV, which are derived from the concepts of chaos and fractal theories, are considered to be a useful tool and have proven valuable in complementing conventional measures in various clinical conditions, ${ }^{10-12,20,21}$ few studies have addressed their usefulness in patients with GAD. In particular, complexity measures have been reported to be useful in uncovering subtle alterations of autonomic neurocardiac modulation and to have utility in broader clinical and research applications. ${ }^{14,22,23}$ Hence, the purpose of the present study was to investigate both linear and nonlinear complexity measures of HRV and to evaluate the relationship between HRV parameters and the severity of anxiety, in medication-free patients with GAD.

\section{Materials and methods Subjects}

The study protocol was approved by the Institutional Review Board of the Gachon University Gil Medical Center, and all procedures used in the study were conducted in accordance with the Declaration of Helsinki. The criteria for patient recruitment were 1) a diagnosis of GAD according to the Diagnostic and Statistical Manual of Mental Disorders, Fourth edition (DSM-IV), ${ }^{1}$ which was established using the Structured Clinical Interview for DSM-IV;24 2) age 20-50 years; 3) not taking psychotropic medication such as antidepressants or benzodiazepines for at least 7 days before the HRV assessment; and 4) not taking any drugs that affect heart rate, such as beta blockers or other antiarrhythmic agents. Patients were excluded if they 1) met diagnostic criteria for a psychiatric diagnosis other than GAD; 2) had a concurrent diagnosis of substance abuse or dependence; 3) had any concurrent cardiovascular, neurological, or endocrinological diseases. Healthy control subjects were also recruited and screened with a complete medical and psychiatric examination, and none had a history of any disease or medication, such as beta blockers or other antiarrhythmic agents, which could have affected the ANS. Written informed consent was obtained after a full explanation on the study procedure.

\section{Assessments}

HRV measurements were performed on the same day following the clinical evaluation. Vigorous exercise and caffeinated beverages, including coffee and tea, were not allowed on the day prior to the HRV measurement. After each subject had been allowed to adapt to the experimental conditions for $\sim 10$ minutes, a 10-minute single channel (three-lead) electrocardiogram (ECG) recording was performed, while the participants were resting in a seated position. The ECG signal was amplified and digitized at a sampling rate of $400 \mathrm{~Hz}$ (width path $0.05-35 \mathrm{~Hz}$ ). The RR interval time series was generated using the automatic scheme in order to detect the $\mathrm{R}$ peak in the ECG, using methods proposed previously. ${ }^{25}$

In the conventional time domain analysis of HRV, the standard deviation of RR intervals (SDNN) and the square root of the mean squared differences of successive normal sinus intervals (RMSSD) were computed, according to the standardized procedures. ${ }^{25} \mathrm{~A}$ spectral analysis was carried out using fast Fourier transformation for the frequency domain analysis. Three major frequency ranges, the very low frequency (VLF) band $(<0.04 \mathrm{~Hz})$, low frequency (LF) band (0.04-0.15 Hz), and high frequency (HF) band $(0.15-0.4 \mathrm{~Hz})$, were calculated, as previously described..$^{25}$

For the nonlinear complexity measure, the approximate entropy (ApEn), which is a parameter developed to quantify the degree of regularity versus the unpredictability in a higher dimensional attractor reconstructed from a time series, such as the instantaneous heart rate time series, was calculated using the method proposed by Pincus. ${ }^{26}$ The ApEn measures the difference between the logarithmic frequencies of similar runs of length " $m$ " and runs with the length " $m+1$ ", calculating the logarithmic likelihood that runs of patterns, which are close to each other, will remain close in the next incremental comparisons. ${ }^{27-31}$ The ApEn is strongly correlated with 
respiratory sinus arrhythmia (RSA). ${ }^{29-31}$ A lower value of ApEn reflects a higher degree of regularity, and conversely the higher the entropy value, the more unpredictable the time series.

The severity of anxiety symptoms in patients with GAD was assessed using the State-Trait Anxiety Inventory $(\mathrm{STAI})^{32}$ and Beck Anxiety Inventory (BAI). ${ }^{33}$

\section{Statistical analysis}

Demographic variables were compared between the groups using $t$-tests and chi-square tests, as appropriate. Since the HRV data in our study did not meet the assumption of normality, the data were log-transformed. However, log transformation also did not normalize the data, other than for the RMSSD value (Kolmogorov-Smirnov $P$-value; SDNN: 0.001, RMSSD: 0.079, VLF: $<0.001$, LF: $<0.001$, HF: 0.002). Therefore, nonparametric Mann-Whitney $U$-tests were used for between-group comparisons, and $P<0.01$ was considered statistically significant with adjustment for multiple comparisons. The level of $P<0.05$ was considered a statistical tendency. The correlations between the HRV measures and the severity of anxiety symptoms in the patient group were assessed using partial correlation analysis, controlling for the effects of age. The research further performed correlation analysis using the locally weighted scatterplot smoothing (LOESS) method to examine a possible curvilinear relationship. In partial correlation analysis, the level of significance was $P<0.01$ with adjustment for multiple correlations. All statistical analyses were performed using SPSS 19.0 for windows (SPSS, Chicago, IL, USA).

\section{Results}

Forty-two outpatients (23 men and 19 women) with GAD and 50 (33 men and 17 women) healthy control subjects were enrolled. The patients had a mean age of $33.8 \pm 7.7$ years and a mean duration of illness of $2.5 \pm 1.3$ years. At enrollment, the mean total STAI-State, STAI-Trait, and BAI scores of the patients were $56.1 \pm 7.6,56.3 \pm 9.1$, and $31.0 \pm 11.5$, respectively. Table 1 presents the demographic characteristics of the groups. There were no significant differences in age, gender, or smoking status between the groups $(P>0.1)$ (Table 1$)$.

A comparison of the HRV parameters between the groups is shown in Table 2. Compared to the control group, the patient group showed significantly lower SDNN ( $z$ score $=-3.77, P<0.01$ ) and $\operatorname{RMSSD}(z$ score $=-2.91$, $P<0.01$ ) values (Table 2 ). There was no significant difference in the power spectrum values between the two groups (VLF: $z$ score $=-1.81, P=0.07$; LF: $z$ score $=-2.33, P=0.02$; HF: $z$ score $=-0.40, P=0.69$ ) (Table 2). The LF and VLF values tended to be lower in the patient group compared to the control group, which did not reach statistical significance. The ApEn value, which is a nonlinear complexity indicator, was significantly lower in the patient group compared to the control group $(z$ score $=-7.61, P<0.01)$ (Table 2$)$.

To explore the correlations between HRV parameters and the severity of anxiety symptoms in the patient group, the research performed partial correlation analyses controlling for age, since it was significantly correlated negatively with some HRV parameters (SDNN: $r=-0.45, P<0.01$; RMSSD: $r=-0.28, P=0.07$; VLF: $r=-0.44, P<0.01$; LF: $r=-0.38$, $P=0.01$; HF: $r=-0.28, P=0.07$; ApEn: $r=0.03, P=0.84)$. The analysis showed that there were no significant correlations between HRV parameters and the severity of anxiety symptoms (Table 3 ). There was a weak correlation between STAIState and RMSSD ( $r=0.33, P=0.04)$, which did not reach statistical significance (Table 3 ). The results of the LOESS regression indicated that RMSSD has a slight increasing trend with a STAI-State score $>60$ (Figure 1).

\section{Discussion}

The present study investigated linear and nonlinear complexity measures of HRV and evaluated the relationship between HRV parameters and the severity of anxiety, in medicationfree patients with GAD. It observed that both time domain

Table I Comparison of demographic variables between the groups

\begin{tabular}{|c|c|c|c|c|}
\hline & $\begin{array}{l}\text { Patients with GAD } \\
(n=42)\end{array}$ & $\begin{array}{l}\text { Control subjects } \\
(n=50)\end{array}$ & $t$ score & $\begin{array}{l}\text { P-value } \\
\text { (two-tailed) }\end{array}$ \\
\hline Age & $33.8(7.7)$ & $32.7(9.2)$ & 0.63 & 0.53 \\
\hline Gender (male/female) & $23 / 19$ & $33 / 17$ & $\chi^{2}=1.21$ & 0.29 \\
\hline Smokers/nonsmokers & $15 / 27$ & $15 / 35$ & $\chi^{2}=0.34$ & 0.66 \\
\hline Duration of illness (year) & $2.5(1.3)$ & NA & NA & NA \\
\hline STAI-State score & $56.1(7.6)$ & NA & NA & NA \\
\hline STAI-Trait score & $56.3(9.1)$ & NA & NA & NA \\
\hline BAl score & $31.0(11.5)$ & NA & NA & NA \\
\hline
\end{tabular}

Note: Values are presented as mean (standard deviation).

Abbreviations: GAD, generalized anxiety disorder; NA, not applicable; STAI, State-Trait Anxiety Inventory; BAI, Beck Anxiety Inventory. 
Table 2 Comparison of HRV measures between the groups

\begin{tabular}{|c|c|c|c|c|}
\hline HRV parameters & $\begin{array}{l}\text { Patients with GAD } \\
(n=42)\end{array}$ & $\begin{array}{l}\text { Control subjects } \\
(n=50)\end{array}$ & z score & $\begin{array}{l}\text { P-value } \\
\text { (two-tailed) }\end{array}$ \\
\hline SDNN & $34.1(14.6)$ & $46.7(15.4)$ & -3.77 & $<0.01 *$ \\
\hline RMSSD & $23.1(12.4)$ & $31.3(15.0)$ & -2.91 & $<0.01 *$ \\
\hline VLF & $480.9(422.8)$ & $605.0(419.9)$ & $-1.8 \mid$ & 0.07 \\
\hline LF & $278.4(257.0)$ & $395.9(319.4)$ & -2.33 & 0.02 \\
\hline $\mathrm{HF}$ & $238.3(367.0)$ & 168.1 (151.5) & -0.40 & 0.69 \\
\hline ApEn & $\mathrm{I} . \mathrm{I}(0.2)$ & $\mathrm{I} .5(0.2)$ & $-7.6 I$ & $<0.01 *$ \\
\hline
\end{tabular}

Notes: Values are presented as mean (standard deviation) and were analyzed using Mann-Whitney $U$-test. $* P<0.01$.

Abbreviations: HRV, heart rate variability; GAD, generalized anxiety disorder; SDNN, the standard deviation of all RR intervals; RMSSD, the square root of the mean squared differences of successive normal sinus intervals; VLF, very low frequency; LF, low frequency; HF, high frequency; ApEn, approximate entropy.

and nonlinear complexity parameters were significantly lower in patients with GAD than in healthy control subjects. The time domain measures, such as SDNN and RMSSD, have been reported to reflect the parasympathetic modulation. ${ }^{25,34}$ In addition, the value of the nonlinear complexity measure, ApEn, has been reported to have a direct relationship with parasympathetic activity. ${ }^{35}$ Therefore, the results suggest that autonomic neurocardiac dysfunction in patients with GAD is best characterized as decreased parasympathetic regulation.

They are in line with previous reports that HRV parameters, reflecting parasympathetic modulation, were significantly reduced in patients with GAD relative to control subjects. ${ }^{15-19}$ In addition, they are consistent with the notion that chronic worry is associated with decreased vagal activity and autonomic inflexibility. ${ }^{36}$

Notably, the nonlinear complexity measure, ApEn, was significantly lower in the patients with GAD compared to the control group. The nonlinear parameters of HRV have not been applied until now to patients with GAD, and this study believes that it exists as the first report to demonstrate the reduction of a nonlinear complexity measure in those patients. Central autonomic regulation induces nonlinear phenomena in sinus rhythm generation. The HRV analysis based on the nonlinear theory has been found to better characterize the multiple regulatory systems influencing the modulation of the heart rate time series in complex biological systems. ${ }^{27}$ The decrease in the entropy value indicates a decrease in the number of the variables and their levels of interactions involved in autonomic neurocardiac regulation. ${ }^{30}$ Therefore, decreased entropy measures in patients with GAD suggest that the degree of distribution of stochastic processes gets lower ${ }^{26}$ and that the neuroautonomic control system governing heart rate loses complexity in these patients. These changes may be significantly associated with the diminished adaptability of the bio-system. ${ }^{30}$ The results of this study suggest the usefulness of nonlinear complexity measures in evaluating autonomic neurocardiac function in GAD.

It is interesting to note that LF and VLF tend to be lower in the patient group compared to the control group. The LF power is considered to reflect both parasympathetic and sympathetic modulation, ${ }^{34}$ In addition, factors including multiple neural reflexes and adrenergic receptor sensitivity have also been suggested to be involved in LF power. ${ }^{37}$ Recent studies ${ }^{38}$ showed that the LF component is predominantly determined by the parasympathetic system. ${ }^{38}$ The origin of VLF power is not entirely clear, although it has been associated with thermoregulation and the renin-angiotensin system. ${ }^{39}$

Table 3 Correlation coefficients between the HRV parameters and the severity of anxiety symptoms in the generalized anxiety disorder group

\begin{tabular}{llll}
\hline HRV parameters & STAI-State score & STAI-Trait score & BAI score \\
\hline SDNN & $0.23(0.16)$ & $0.10(0.57)$ & $0.13(0.42)$ \\
RMSSD & $0.33(0.04)$ & $0.23(0.17)$ & $0.25(0.13)$ \\
VLF & $0.15(0.38)$ & $-0.04(0.83)$ & $-0.01(0.97)$ \\
LF & $-0.02(0.92)$ & $-0.09(0.59)$ & $0.15(0.36)$ \\
HF & $0.22(0.19)$ & $0.10(0.55)$ & $0.11(0.51)$ \\
ApEn & $0.02(0.92)$ & $-0.04(0.82)$ & $0.04(0.83)$ \\
\hline
\end{tabular}

Note: The $P$-values are presented in parentheses.

Abbreviations: HRV, heart rate variability; STAI, State-Trait Anxiety Inventory; BAI, Beck Anxiety Inventory; SDNN, the standard deviation of all RR intervals; RMSSD, the square root of the mean squared differences of successive normal sinus intervals; VLF, very low frequency; LF, low frequency; HF, high frequency; ApEn, approximate entropy. 


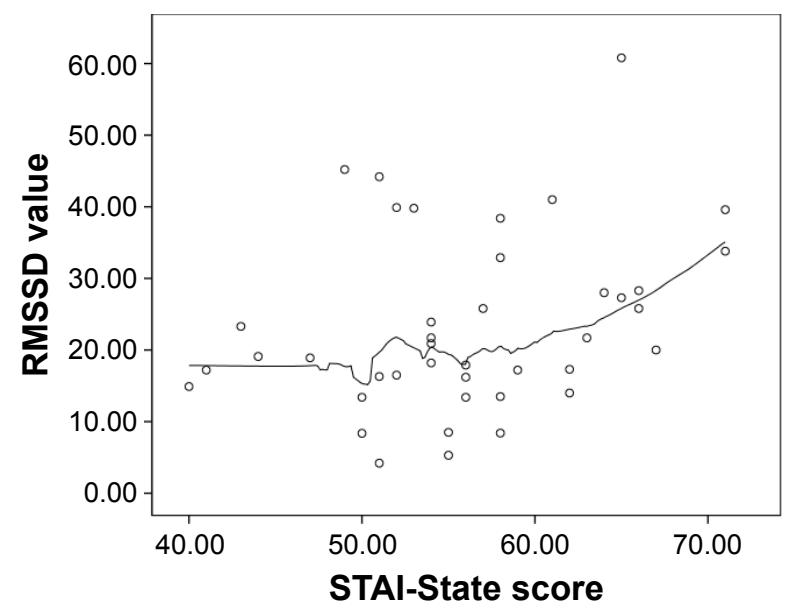

Figure I Scatter plots of STAI-State score and RMSSD value in the patient group. The line in the figure represents a LOESS curve fitted to the values.

Abbreviations: STAI, State-Trait Anxiety Inventory; RMSSD, the square root of the mean squared differences of successive normal sinus intervals; LOESS, locally weighted scatterplot smoothing.

Recent studies have suggested the significant influence of parasympathetic outflow, ${ }^{39,40}$ implying the critical importance of parasympathetic mechanisms in the derivation of the VLF value. Therefore, the results for the LF and VLF values are another indication that GAD is associated with decreased parasympathetic regulation.

It should be noted that RSA is one of the most studied and frequently used HRV measures. The RSA is considered to be an index of cardiac vagal tone. ${ }^{41}$ However, it has the limitation of being potentially confounded by individual differences in respiratory behavior. ${ }^{42,43}$ In this study, SDNN, RMSSD, and frequency domain measures were used, which have been found to be significantly related to RSA. ${ }^{44} 46$ Therefore, this study is clearly in line with previous studies using RSA, in terms of the HRV index for parasympathetic (vagal) tone, and the results of this study clearly suggest diminished vagal tone in patients with GAD.

Recent studies have shown that GAD is associated with the dysfunction of a wide cortical and subcortical network involving prefrontal cortex, anterior cingulate cortex, amygdala, and the bed nucleus of the stria terminalis. ${ }^{47,48}$ Considering that this neural circuit is a crucial part of the central autonomic network, ${ }^{49}$ it is plausible that HRV parameters might reflect the functional activity of this neural circuit, which could manifest phenotypically as chronic anxiety and worry. Moreover, the HRV has been used for the identification of autonomic dysfunctions in noncardiac patients on the assumption that, if such a dysfunction is identified, it is an indication of a more widespread neuroautonomic dysregulation. ${ }^{50}$ In this context, the results of this study may imply the usefulness of HRV indices as objective quantitative measures of GAD.

On the other hand, reduced HRV, especially diminished parasympathetic activity, has been found in schizophrenia, ${ }^{23}$ bipolar mania, ${ }^{51}$ and depression ${ }^{52}$ and is presumably associated with inherent characteristics of the illnesses, independent of the effects of psychotropic medications. Therefore, reduced HRV itself may not be a specific marker for GAD, but the results of this study further verify significantly altered heart rate dynamics in psychiatric disorders. Moreover, mental stress is known to reduce cardiac parasympathetic tone, and thus it has been associated with reduced HRV. ${ }^{53-56}$ In particular, patients with psychiatric disorders associated with emotional symptoms are likely to experience greater subjective distress or perceived threat. In this context, reduced HRV might precede the onset and clinical diagnosis of GAD and might be a trait or vulnerability marker of the risk of developing a psychiatric disorder, especially one of an affective nature.

In the investigation of the biological markers for GAD, psychiatric comorbidity can be one of the confounding factors. Previous studies ${ }^{18,19}$ have reported that GAD has a high rate of comorbidity with other psychiatric disorders, such as major depression, bipolar disorder, and other anxiety disorders. A recent study showed that patients with GAD and comorbid depression had the greatest reductions in HRV among the three groups of GAD without comorbidity, GAD with comorbid major depression, and healthy controls, suggesting a possible cumulative effect. ${ }^{18}$ In this study, patients were excluded if they met diagnostic criteria for a psychiatric diagnosis other than GAD. However, since this study did not evaluate the severity of depressive symptoms or the lifetime comorbidity of depression, future studies should include comorbid patients in order to address the cumulative effect or to elucidate whether GAD patients with comorbid depression have distinctive neurocardiac characteristics in comparison to non-comorbid patients.

In correlation analysis, there were no significant correlations between HRV parameters and the severity of anxiety symptoms, which indicates that there was no significant relationship between them in there subjects. This study further performed correlation analysis using LOESS, and the results of the LOESS regression indicated that RMSSD has an increasing trend with a STAI-State score $>60$, suggesting possible compensatory regulation of the parasympathetic nervous system in response to the increased severity of anxiety. Further studies with a larger sample are required to investigate the relationship between various linear and 
nonlinear HRV measures and clinical severity in patients with GAD.

It should be noted that the patients were medication-free at the time of enrollment. A previous study reported that anxiety disorders are associated with significantly lower HRV but that the association is mainly driven by the effects of antidepressants, such as tricyclic antidepressants and selective serotonin reuptake inhibitors, ${ }^{43,57}$ which decrease vagal activity and alter vagal outflow. ${ }^{19,57,58}$ Previous studies have also shown that benzodiazepines can influence autonomic neurocardiac regulation, reducing central vagal tone, possibly through their interaction with the gamma-aminobutyric acid-A receptor chloride ion channel complex. ${ }^{59}$ However, there have been other studies reporting that medicationfree patients with GAD show significantly reduced HRV measures and that GAD is associated with reduced HRV regardless of the effects of antidepressants. ${ }^{16,18,19}$ Although, in this study, patients were all medication-free for at least 7 days at the time of HRV assessment, the after-effects of previous pharmacotherapy might have affected the values of the HRV parameters.

The interpretation of the results should be considered in light of some limitations. The study did not measure body mass index, which was reported to be one of the factors influencing baroreflex sensitivity. ${ }^{60}$ Consumption of coffee or tea was not allowed on the day prior to the HRV measurement. However, the study did not measure the daily average amount of coffee or tea intake, which might have affected autonomic regulation. ${ }^{61}$ The severity of anxiety symptoms was assessed using STAI-State, STAI-Trait, and BAI. Further investigation implementing interviewer-rated scales may be required. In addition, future prospective studies are required to investigate the effects of pharmacological or nonpharmacological treatment on neuroautonomic modulation in patients with GAD. ${ }^{6}$ In this study, a 10-minute single channel (three-lead) ECG recording was performed. The HRV indices obtained from ambulatory 24-hour ECG recordings are more accurate on reflection of daily activities. A short acquisition in the hospital can confound findings due to the stress of being examined. However, a short-term HRV measurement (5-15 minutes), which is made under controlled conditions, has practical advantages and reproducibility. ${ }^{62,63}$ In addition, it also provides almost immediate measurement results and enables real-time monitoring of ANS function, exhibiting physiological and pathological changes in the ANS. ${ }^{62-64}$ Moreover, previous studies on GAD also used short-term HRV measurements. ${ }^{18,19}$

\section{Conclusion}

In conclusion, the present study indicates that GAD is significantly associated with reduced HRV, suggesting that autonomic neurocardiac integrity is substantially impaired in patients with GAD. The results of the present study also indicate that both linear and nonlinear complexity measures of HRV may have utility in evaluating neuroautonomic function in patients with GAD.

\section{Acknowledgments}

This work was supported by a grant of the Korean Health Technology R\&D Project, Ministry of Health \& Welfare, Republic of Korea (HI14C1731) and by the Gachon University Research Fund of 2015 (GCU-2015-5031). This work was also supported in part by the Brain Research Program through the National Research Foundation of Korea (NRF) funded by the Ministry of Science, ICT \& Future Planning (2016M3C7A1914451).

\section{Disclosure}

The authors report no conflicts of interest in this work.

\section{References}

1. American Psychiatric Association. Diagnostic and Statistical Manual of Mental Disorders. 4th ed. Washington DC: American Psychiatric Press; 1994.

2. Miloyan B, Pachana NA. Clinical significance of worry and physical symptoms in late-life generalized anxiety disorder. Int J Geriatr Psychiatry. 2015;30(12):1186-1194.

3. Tully PJ, Cosh SM, Baune BT. A review of the affects of worry and generalized anxiety disorder upon cardiovascular health and coronary heart disease. Psychol Health Med. 2013;18(6):627-644.

4. Jetty PV, Charney DS, Goddard AW. Neurobiology of generalized anxiety disorder. Psychiatr Clin North Am. 2001;24(1):75-97.

5. Fisher AJ, Newman MG. Heart rate and autonomic response to stress after experimental induction of worry versus relaxation in healthy, high-worry, and generalized anxiety disorder individuals. Biol Psychol. 2013;93(1):65-74.

6. Kalk NJ, Nutt DJ, Lingford-Hughes AR. The role of central noradrenergic dysregulation in anxiety disorders: evidence from clinical studies. J Psychopharmacol. 2011;25(1):3-16.

7. Huikuri HV, Kessler KM, Terracall E, Castellanos A, Linnaluoto MK, Myerburg RJ. Reproducibility and circadian rhythm of heart rate variability in healthy subjects. Am J Cardiol. 1990;65(5):391-393.

8. Yeragani VK. Heart rate and blood pressure variability: implications for psychiatric research. Neuropsychobiology. 1995;32(4):182-191.

9. Chalmers JA, Quintana DS, Abbott MJ, Kemp AH. Anxiety disorders are associated with reduced heart rate variability: a meta-analysis. Front Psychiatry. 2014;(5):80.

10. Haapaniemi TH, Pursiainen V, Korpelainen JT, Huikuri HV, Sotaniemi KA, Myllylä VV. Ambulatory ECG and analysis of heart rate variability in Parkinson's disease. J Neurol Neurosurg Psychiatry. 2001; 70(3);305-310.

11. Ansakorpi H, Korpelainen JT, Huikuri HV, Tolonen U, Myllylä VV, Isojärvi JI. Heart rate dynamics in refractory and well controlled temporal lobe epilepsy. J Neurol Neurosurg Psychiatry. 2002;72(1):26-30. 
12. Yeragani VK, Nadella R, Hinze B, Yeragani S, Jampala VC. Nonlinear measures of heart period variability: decreased measures of symbolic dynamics in patients with panic disorder. Depress Anxiety. 2000;12(2): 67-77.

13. Bär KJ, Koschke M, Berger S, et al. Influence of olanzapine on QT variability and complexity measures of heart rate in patients with schizophrenia. J Clin Psychopharmacol. 2008;28(6):694-698.

14. Kim JH, Yi SH, Lee J, Kim YS. Effects of clozapine on heart rate dynamics and their relationship with therapeutic response in treatmentresistant schizophrenia. J Clin Psychopharmacol. 2013;33(1):69-73.

15. Kollai M, Kollai B. Cardiac vagal tone in generalised anxiety disorder. Br J Psychiatry. 1992;161:831-835.

16. Thayer JF, Friedman BH, Borkovec TD. Autonomic characteristics of generalized anxiety disorder and worry. Biol Psychiatry. 1996;39(4): 255-266.

17. Pittig A, Arch JJ, Lam CW, Craske MG. Heart rate and heart rate variability in panic, social anxiety, obsessive-compulsive, and generalized anxiety disorders at baseline and in response to relaxation and hyperventilation. Int J Psychophysiol. 2013;87(1):19-27.

18. Chang HA, Chang CC, Tzeng NS, Kuo TB, Lu RB, Huang SY. Generalized anxiety disorder, comorbid major depression and heart rate variability: a case-control study in Taiwan. Psychiatry Investig. 2013;10(4): 326-335.

19. Kemp AH, Brunoni AR, Santos IS, et al. Effects of depression, anxiety, comorbidity, and antidepressants on resting-state heart rate and its variability: an ELSA-Brasil cohort baseline study. Am J Psychiatry. 2014;171(12):1328-1334.

20. Kim JH, Yi SH, Yoo CS, et al. Heart rate dynamics and their relationship to psychotic symptom severity in clozapine-treated schizophrenic subjects. Prog Neuropsychopharmacol Biol Psychiatry. 2004; 28(2):371-378.

21. Tang SC, Jen HI, Lin YH, et al. Complexity of heart rate variability predicts outcome in intensive care unit admitted patients with acute stroke. J Neurol Neurosurg Psychiatry. 2015;86(1):95-100.

22. Goldberger AL, Amaral LA, Hausdorff JM, Ivanov PCh, Peng CK Stanley HE. Fractal dynamics in physiology: alterations with disease and aging. Proc Natl Acad Sci U S A. 2002;99(Suppl 1):2466-2472.

23. Bär KJ, Boettger MK, Koschke M, et al. Non-linear complexity measures of heart rate variability in acute schizophrenia. Clin Neurophysiol. 2007;118(9):2009-2015.

24. First MB, Spitzer RL, Gibbon M, Williams JBW. Structured Clinical Interview for DSM-IV Axis I Disorders Research Version (SCID-I). New York State Psychiatric Institute: Biometrics Research; 1996.

25. Task Force of the European Society of Cardiology and the North American Society of Pacing and Electrophysiology. Heart rate variability: standards of measurement, physiological interpretation and clinical use. Circulation. 1996;93(5):1043-1065.

26. Pincus SM. Approximate entropy as a measure of system complexity. Proc Natl Acad Sci U S A. 1991;88(6):2297-2301.

27. Seely AJ, Macklem PT. Complex systems and the technology of variability analysis. Crit Care. 2004;8(6):R367-R384.

28. Yeragani VK, Pohl R, Mallavarapu M, Balon R. Approximate entropy of symptoms of mood: an effective technique to quantify regularity of mood. Bipolar Disord. 2003;5(4):279-286.

29. Yeragani VK, Sobolewski E, Kay J, Jampala VC, Igel G. Effect of age on long-term heart rate variability. Cardiovasc Res. 1997;35(1):35-42.

30. Yeragani VK, Sobolewski E, Jampala VC, Kay J, Yeragani S, Igel G. Fractal dimension and approximate entropy of heart period and heart rate: awake versus sleep differences and methodological issues. Clin Sci (Lond). 1998;95(3):295-301.

31. Richman JS, Moorman JR. Physiological time-series analysis using approximate entropy and sample entropy. Am J Physiol Heart Circ Physiol. 2000;278(6):H2039-H2049.

32. Spielberger CD, Gorsuch RL, Lushene R, Vagg PR, Jacobs GA. Manual for the State-Trait Anxiety Inventory. Palo Alto, CA: Consulting psychologists Press; 1983.
33. Beck AT, Steer RA. Beck Anxiety Inventory Manual. San Antonio: The Psychological Corporation Harcourt; 1990.

34. Mueck-Weymann M, Rechlin T, Ehrengut F, et al. Effects of olanzapine and clozapine upon pulse rate variability. Depress Anxiety. 2002; 16(3):93-99.

35. Bolea J, Pueyo E, Laguna P, Bailón R. Non-linear HRV indices under autonomic nervous system blockade. Conf Proc IEEE Eng Med Biol Soc. 2014;2014:3252-3255.

36. Hammel JC, Smitherman TA, McGlynn FD, Mulfinger AM, Lazarte AA, Gothard KD. Vagal influence during worry and cognitive challenge. Anxiety Stress Coping. 2011;24(2):121-136.

37. Kingwell BA, Thompson JM, Kaye DM, McPherson GA, Jennings GL, Esler MD. Heart rate spectral analysis, cardiac norepinephrine spillover, and muscle sympathetic nerve activity during human sympathetic nervous activation and failure. Circulation. 1994;90(1):234-240.

38. Reyes del Paso GA, Langewitz W, Mulder LJ, van Roon A, Duschek S. The utility of low frequency heart rate variability as an index of sympathetic cardiac tone: a review with emphasis on a reanalysis of previous studies. Psychophysiology. 2013;50(5):477-487.

39. Tripathi KK. Very low frequency oscillations in the power spectra of heart rate variability during dry supine immersion and exposure to non-hypoxic hypobaria. Physiol Meas. 2011;32(6):717-729.

40. Taylor JA, Carr DL, Myers CW, Eckberg DL. Mechanisms underlying very-low-frequency RR-interval oscillations in humans. Circulation. 1998;98(6):547-555.

41. Lane JD, Adcock RA, Burnett RE. Respiratory sinus arrhythmia and cardiovascular responses to stress. Psychophysiology. 1992;29(4): $461-470$.

42. Grossman P, Kollai M. Respiratory sinus arrhythmia, cardiac vagal tone, and respiration: within- and between-individual relations. Psychophysiology. 1993;30(5):486-495.

43. Licht CM, de Geus EJ, Zitman FG, Hoogendijk WJ, van Dyck R, Penninx BW. Association between major depressive disorder and heart rate variability in the Netherlands Study of Depression and Anxiety (NESDA). Arch Gen Psychiatry. 2008;65(12):1358-1367.

44. Vieira CF, Lima MM, Costa HS, et al. Correlation between indexes of autonomic maneuvers and heart rate variability in hemodialysis patients. Clin Auton Res. 2016;26(3):181-188.

45. Smith AL, Owen H, Reynolds KJ. Heart rate variability indices for very short-term (30 beat) analysis. Part 2: validation. J Clin Monit Comput. 2013;27(5):577-585.

46. van den Berg MP, Haaksma J, Brouwer J, Tieleman RG, Mulder G, Crijns HJ. Heart rate variability in patients with atrial fibrillation is related to vagal tone. Circulation. 1997;96(4):1209-1216.

47. Yassa MA, Hazlett RL, Stark CE, Hoehn-Saric R. Functional MRI of the amygdala and bed nucleus of the stria terminalis during conditions of uncertainty in generalized anxiety disorder. J Psychiatr Res. 2012;46(8):1045-1052.

48. Robinson OJ, Krimsky M, Lieberman L, Allen P, Vytal K, Grillon C. Towards a mechanistic understanding of pathological anxiety: the dorsal medial prefrontal-amygdala 'aversive amplification' circuit in unmedicated generalized and social anxiety disorders. Lancet Psychiatry. 2014;1(4):294-302.

49. Benarroch EE. The central autonomic network: functional organization, dysfunction, and perspective. Mayo Clin Proc. 1993;68(10) 988-1001.

50. Malik M, Camm AJ, editors. Heart Rate Variability. Armonk (NY): Futura Publishing Company; 1995.

51. Henry BL, Minassian A, Paulus MP, Geyer MA, Perry W. Heart rate variability in bipolar mania and schizophrenia. J Psychiatr Res. 2010 ; 44(3):168-176.

52. Stapelberg NJ, Hamilton-Craig I, Neumann DL, Shum DH, McConnell $\mathrm{H}$. Mind and heart: heart rate variability in major depressive disorder and coronary heart disease - a review and recommendations. Aust N Z J Psychiatry. 2012;46(10):946-957. 
53. Berntson GG, Cacioppo JT, Quigley KS. Respiratory sinus arrhythmia: autonomic origins, physiological mechanisms, and psychophysiological implications. Psychophysiology. 1993;30(2):183-196.

54. Porges SW. Cardiac vagal tone: a physiological index of stress. Neurosci Biobehav Rev. 1995;19(2):225-233.

55. Hjortskov N, Rissén D, Blangsted AK, Fallentin N, Lundberg U, Søgaard K. The effect of mental stress on heart rate variability and blood pressure during computer work. Eur J Appl Physiol. 2004;92(1-2): 84-89.

56. Thayer JF, Ahs F, Fredrikson M, Sollers JJ 3rd, Wager TD. A metaanalysis of heart rate variability and neuroimaging studies: implications for heart rate variability as a marker of stress and health. Neurosci Biobehav Rev. 2012;36(2):747-756.

57. Licht CM, de Geus EJ, van Dyck R, Penninx BW. Association between anxiety disorders and heart rate variability in The Netherlands Study of Depression and Anxiety (NESDA). Psychosom Med. 2009; 71(5):508-518.

58. Fisher AJ, Woodward SH. Cardiac stability at differing levels of temporal analysis in panic disorder, post-traumatic stress disorder, and healthy controls. Psychophysiology. 2014;51(1):80-87.
59. Agelink MW, Majewski TB, Andrich J, Mueck-Weymann M. Shortterm effects of intravenous benzodiazepines on autonomic neurocardiac regulation in humans: a comparison between midazolam, diazepam, and lorazepam. Crit Care Med. 2002;30(5):997-1006.

60. Skrapari I, Tentolouris N, Perrea D, Bakoyiannis C, Papazafiropoulou A, Katsilambros N. Baroreflex sensitivity in obesity: relationship with cardiac autonomic nervous system activity. Obesity (Silver Spring). 2007;15(7):1685-1693.

61. Yeragani VK, Krishnan S, Engels HJ, Gretebeck R. Effects of caffeine on linear and nonlinear measures of heart rate variability before and after exercise. Depress Anxiety. 2005;21(3):130-134.

62. Kleiger RE, Stein PK, Bigger JT Jr. Heart rate variability: measurement and clinical utility. Ann Noninvasive Electrocardiol. 2005;10(1): 88-101.

63. Min KB, Min JY, Paek D, Cho SI, Son M. Is 5-minute heart rate variability a useful measure for monitoring the autonomic nervous system of workers? Int Heart J. 2008;49(2):175-181.

64. Ji L, Li P, Li K, Wang X, Liu C. Analysis of short-term heart rate and diastolic period variability using a refined fuzzy entropy method. Biomed Eng Online. 2015;14:64
Neuropsychiatric Disease and Treatment

\section{Publish your work in this journal}

Neuropsychiatric Disease and Treatment is an international, peerreviewed journal of clinical therapeutics and pharmacology focusing on concise rapid reporting of clinical or pre-clinical studies on a range of neuropsychiatric and neurological disorders. This journal is indexed on PubMed Central, the 'PsycINFO' database and CAS,

\section{Dovepress}

and is the official journal of The International Neuropsychiatric Association (INA). The manuscript management system is completely online and includes a very quick and fair peer-review system, which is all easy to use. Visit http://www.dovepress.com/testimonials.php to read real quotes from published authors. 